\title{
Effect of Cement Base Thicknesses on MOD Amalgam Restorations
}

J. W. FARAH, A. E. CLARK, ${ }^{*}$ M. MOHSEIN, ${ }^{\dagger}$ and P. A. THOMAS

Department of Dental Materials, School of Dentistry, University of Michigan, Ann Arbor, Michigan 48109, ${ }^{*}$ University of Florida, Gainesville, Florida 32610, †University of Alexandria Dental School, Alexandria, Egypt, and $\$$ Department of Biomaterials, University of Florida, Gainesville, Florida 32610

The effect of five cement bases on the fracture strength of three amalgams was determined at $24 \mathrm{~h}$ after condensation. It was found that the type of base used was the most important factor in affecting the fracture strength of the amalgam, followed by the thickness of the base, and finally the type of amalgam that was used.

\section{J Dent Res 62(2):109-111, February 1983}

\section{Introduction.}

The placement of intermediary cement bases under deep preparations for amalgam restorations is a recommended procedure in restorative dentistry.

The base is of low thermal conductivity and thus protects the pulp from thermal shock. ${ }^{1}$ Bases should also possess sufficient strength to withstand the forces of condensation and mastication transmitted through the amalgam restoration. 2,3

The effect of the thickness of various cement bases on the fracture strength of a class II amalgam restoration was recently reported. 4 The study stressed the importance of the modulus of elasticity of cements under amalgam restorations. This study examines the effect of the thickness of five cement bases on three types of amalgams in MOD preparations.

\section{Materials and methods.}

Extracted molar teeth were mounted in clear polyester casting resin. The resin base was $12 \mathrm{~mm}$ in height and from 1 to $2 \mathrm{~mm}$ below the cemento-enamel junction. The occlusal surface was ground flat, and an MOD cavity preparation was cut using a conventional high-speed handpiece. The occlusal-pulpal depth of the preparation was 3.0 $\mathrm{mm}$, as shown in Fig. 1.

Five cements were used as bases of varying thicknesses: (1) calcium hydroxide (Dycal); $\$$ (2) reinforced zinc-oxide eugenol (IRM); ; (3) polycarboxylate (Tylok); $\$$ (4) zinc phosphate (Tenacin); $\$$ and (5) glass ionomer cement (Chembond). $\S$ Conventional (Velvalloy),** high-copper unicompositional (Tytin), ${ }^{* *}$ as well as high-copper admixed (Dispersalloy) \# amalgams were condensed on each of the cement bases.

The thickness of each cement base was varied from 0 $\mathrm{mm}$ (no cement base) to $3.0 \mathrm{~mm}$ (no amalgam) by $0.5-\mathrm{mm}$ increments. Four molar teeth were used for each $0.5-\mathrm{mm}$ increment of cement base. This was repeated for each cement base and each of three types of amalgams.

Received for publication April 19, 1982

Accepted for publication September 13, 1982

Presented at the annual meeting of the American Association for Dental Research, March 19-22, 1981, in Chicago, IL

Dr. Farah acknowledges the following companies for supplying the commercial products: L. D. Caulk, S. S. White, and Johnson \& Johnson.

$\S$ L. D. Caulk Co., Milford, DE 19963

**s. S. White Co., Philadelphia, PA 19102

\#Johnson \& Johnson, E. Windsor, NJ 08520
The preparation of each specimen was similar to that described earlier for Class I restorations. ${ }^{5}$

The completed restorations were tested at $24 \mathrm{~h}$ after amalgam condensation on a universal testing machine in compression at a rate of $0.2 \mathrm{~mm} / \mathrm{min}$. The load was applied in the center of the MOD restoration, mesio-distally, as well as bucco-lingually. In each case, the load at which the amalgam restoration fractured was recorded, and the mean was plotted as a function of the cement base supporting it. A three-way analysis of variance ${ }^{6}$ was carried out to determine the effect of the base, the thickness, and the amalgam on the fracture strength. Tukey's interval for comparisons among means was calculated at the $95 \%$ level of confidence. ${ }^{7}$

\section{Results.}

The fracture load was plotted as a function of the thickness of the various cement bases supporting the conventional, the high-copper admixed, and the high-copper unicompositional amalgam restorations in Figs. 2, 3, and 4, respectively. All specimens were subjected to loading $24 \mathrm{~h}$ after condensation of the amalgam, and the plotted value is a mean of four specimens.

For each amalgam, a decrease in the fracture load occurred as the thickness of the base was increased, and the modulus of the base decreased. This was especially true when marked differences in modulus existed among cements, such as calcium hydroxide, reinforced $Z O E$, and zinc phosphate. The differences were not as clear among cements like zinc phosphate, carboxylate, and glass ionomer.

Model 1125, Instron Corporation, Canton, MA 02021
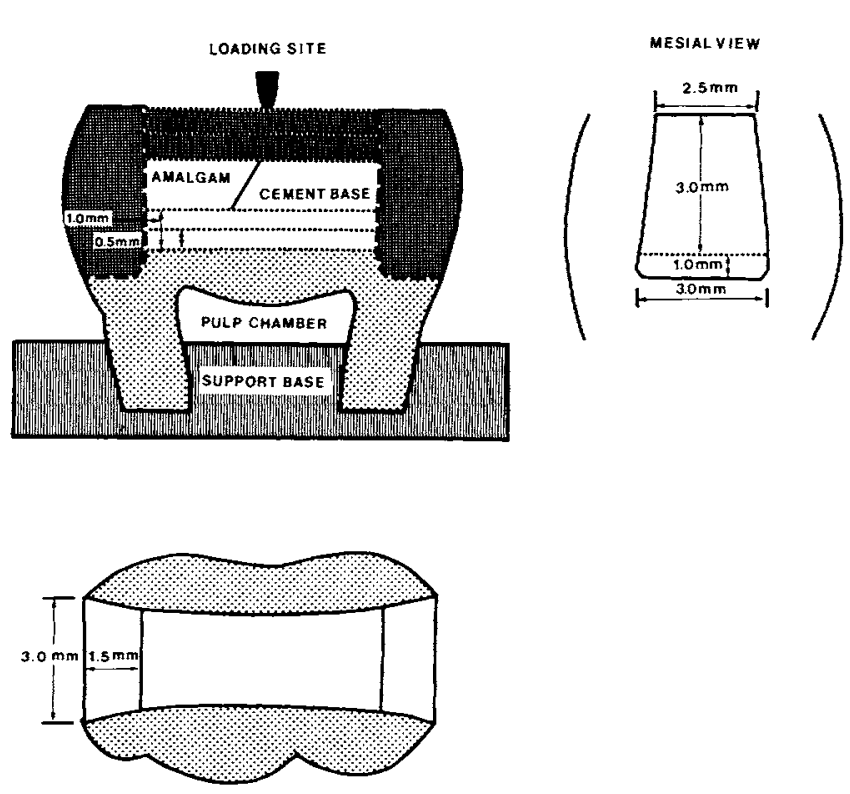

Fig. 1 - Mounted molar with prepared MOD cavity. 


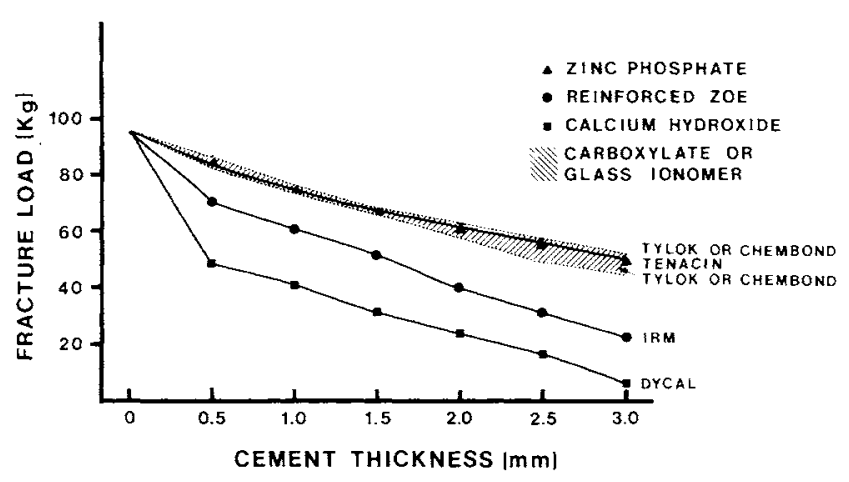

Fig. 2 - Effect of cement bases on Velvalloy in an MOD preparation $24 \mathrm{~h}$ after condensation.

\section{Discussion.}

The use of a cement base is especially recommended in areas that are in close proximity to the pulp. The cement protects the pulp from thermal shock, and, depending on its chemical make-up, it can serve as an obtundent or can stimulate the formation of secondary dentin. Unfortunately, a base can also adversely affect the fracture strength of an amalgam restoration.

As demonstrated in this study, a decrease in fracture strength ensued in each specimen where a base was used. Similar results were reported by Farah et al. ${ }^{5}$ on a Class I cavity preparation. In a Class I cavity preparation, the amalgam is supported almost exclusively by the cement base. In an MOD preparation, the support is derived from the amalgam which rests on the dentin of the proximalgingival floor and that which straddles the cement base.

The effect of a particular base and its thickness on a conventional amalgam is shown in Fig. 2. The strength of the amalgam is most dramatically affected by calcium hydroxide. The fracture strength drops from $95 \mathrm{~kg}$ to $48 \mathrm{~kg}$ when $0.5 \mathrm{~mm}$ of Dycal is added. A more gradual decrease takes place as the thickness of the cement is further increased by $0.5-\mathrm{mm}$ intervals. It is recognized that Dycal is usually recommended for use in thicknesses of $0.5 \mathrm{~mm}$ or less. The remaining thicknesses of Dycal were used for comparative purposes.

A similar trend was observed when a reinforced $\mathrm{ZOE}$ was used as a base. When $0.5 \mathrm{~mm}$ IRM was added, the fracture strength decreased by about $26 \%$, as compared to almost a $50 \%$ drop with the $0.5 \mathrm{~mm}$ Dycal. Addition of a zinc phosphate base under the amalgam had less of an effect on the strength than did either the Dycal or the IRM. No significant differences were observed among the zinc phosphate, the carboxylate, and the glass ionomer cements. The shaded area in Figs. 2, 3, and 4 outlines the range within which the amalgam fractured when supported by the carboxylate or the glass ionomer cement.

The fracture strength of the admixed amalgam, such as Dispersalloy, was similar to the conventional amalgam, as observed in Fig. 3. The fracture strength for the unicompositional high-copper amalgam, such as Tytin, decreased dramatically when $0.5 \mathrm{~mm}$ Dycal was introduced (Fig. 4). The fracture strength of the amalgam decreased from 101 to $38 \mathrm{~kg}$, or about a $60 \%$ drop in strength. As noted previously, a more moderate decrease took place when $0.5 \mathrm{~mm}$

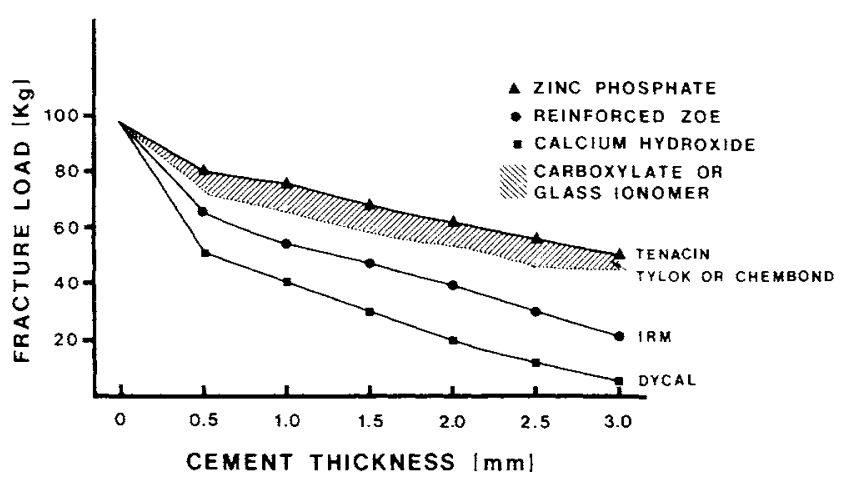

Fig. 3 - Effect of cement bases on Dispersalloy in an MOD preparation $24 \mathrm{~h}$ after condensation.

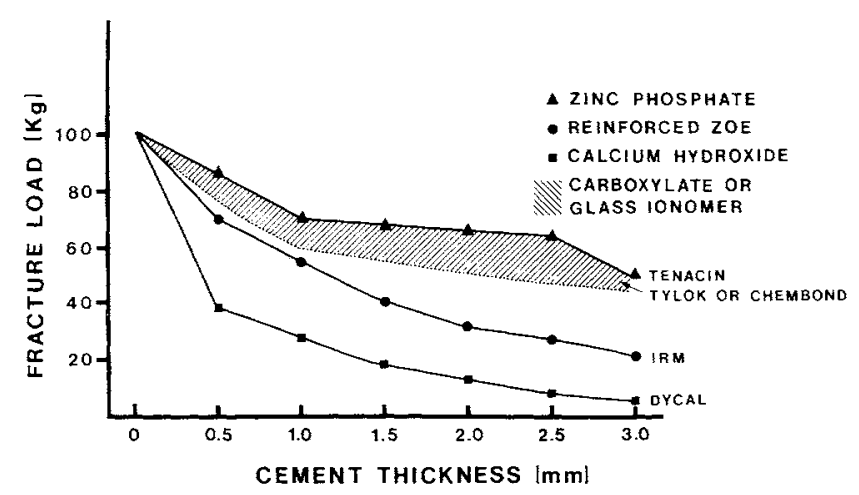

Fig. 4 - Effect of cement bases on Tytin in an MOD preparation $24 \mathrm{~h}$ after condensation.

IRM was used for a base. A similar trend was observed when zinc phosphate was used as a base. Slightly lower fracture strengths were obtained when carboxylate or glass ionomer cements were compared to zinc phosphate cement as shown by the shaded area in Fig. 4. For both the admixed and the unicompositional high-copper amalgam (Figs. 3 and 4), the zinc phosphate cement was consistently the base which resulted in higher fracture strength of the amalgam. Furthermore, less variation from specimen to specimen was observed with the zinc phosphate. Although great care was taken in mixing and proportioning the cements, it was observed that generally a more consistent and bubble-free mix was obtained with zinc phosphate when compared to carboxylate and glass ionomer cement.

All three amalgams (Figs. 2, 3, and 4) exhibited similar fracture trends when supported by respective cement bases. The modulus, which is a measure of rigidity, plays an important role in the supporting ability of the cement base. $^{3}$ Calcium hydroxide has the lowest modulus ${ }^{8}(370$ $\mathrm{MN} / \mathrm{m}^{2}$ ), while zinc phosphate has the highest at 22,000 $\mathrm{MN} / \mathrm{m}^{2}$. Reinforced zinc-oxide eugenol is about $3500 \mathrm{MN}$ / $\mathrm{m}^{2}$, while glass ionomer $l$ and carboxylate cements are about $5000 \mathrm{MN} / \mathrm{m}^{2}$. It is apparent from Figs. 2, 3, and 4 that a cement with a modulus of $5000 \mathrm{MN} / \mathrm{m}^{2}$ or more is

I Personal communication: Dr. Robert G. Craig 
adequate to support an MOD amalgam restoration. This is true because, as mentioned earlier, a good part of the support in an MOD restoration is derived from the amalgam which rests interproximally on the dentin and straddles the cement. In a Class I cavity preparation, the amalgam is almost completely resting on the cement. In such a case, higher moduli values are more beneficial. 5

Another material constant, Poisson's ratio (v), which varies from 0.25 to 0.5 , can also affect, to a more limited degree, the behavior of the cement base, as well as the amalgam restoration. The more brittle materials will have Poisson's values closer to 0.25 , while the more rubbery ones will be closer to 0.5 . Exact values for Poisson's ratio for cements are not available in the literature.

In addition to the modulus and Poisson's ratio, creep seems to play an important role in the fracture of the amalgam. High-copper amalgams, which have lower creep values, tended to have more variation in the fracture data. In other words, their ability to absorb some of the energy was minimal, while the conventional amalgams with the higher creep tended to deform under load and thus attain a more uniform fracture load. Thus, although high-copper amalgams possess higher moduli of elasticity $\left(3.45 \times 10^{4}\right.$ $\left.\mathrm{MN} / \mathrm{m}^{2}\right)$, as compared to conventional amalgams $(2.31 \mathrm{x}$ $\left.10^{4} \mathrm{MN} / \mathrm{m}^{2}\right),{ }^{9}$ the creep value being lower for the former can result in the alloy fracturing at a lower load. This phenomenon was observed in some of the specimens, and one might thus question whether very low creep values are desirable. For example, an amalgam restoration in hyperocclusion could more readily fracture if it could not dissipate some of the energy, i.e., have lower creep.

Roberts et al. ${ }^{10}$ examined the fracture toughness of five amalgams and found that high-copper amalgam alloys exhibited lower toughness values than did conventional amalgam alloys. Thus, one should recognize that the fracture of the amalgam is dependent not only on the modulus and Poisson's ratio of the amalgam and the base, but also on the creep and fracture toughness of the amalgam.

\section{Conclusions.}

1. Addition of $0.5 \mathrm{~mm}$ Dycal base decreased the fracture strength of amalgam by about $50 \%$.
2. Addition of $0.5 \mathrm{~mm}$ IRM base decreased the fracture strength of amalgam by about $25 \%$.

3 . The type of base used played the most important role in affecting the fracture strength of the amalgam.

4. The thickness of the base was the second most important factor which affected the fracture strength of the amalgam.

5. The type of amalgam used played the least important role in the fracture strength of amalgam.

6. The fracture strength of the amalgam was dependent not only on the modulus and Poisson's ratio of the amalgam and/or the cement, but also on the fracture toughness and creep of the amalgam and cement.

\section{REFERENCES}

1. BRADEN, M.: Heat Conduction in Teeth and the Effect of Lining Materials, $J$ Dent Res 43:315, 1964

2. HOPPENSTAND, D.C. and McCONNELL, D.: Mechanical Failure of Amalgam Restorations with Zinc Phosphate and Zinc Oxide Eugenol Cement Bases, $J$ Dent Res 39:899, 1960.

3. FARAH, J.W.; HOOD, J.A.A.; and CRAIG, R.G.: Effect of Cement Bases on the Stresses in Amalgam Restorations, $J$ Dent Res 54:10,1975.

4. HORMATI, A.A. and FULLER, J.L.: The Fracture Strength of Amalgam Overlying Base Materials, J Prosthet Dent 43:52, 1980.

5. FARAH, J.W.; CLARK, A.E.; THOMAS, P.A.; and BORKOW, I.: Cement Bases Under Amalgam Restorations: Effect of Thickness, J Oper Dent 6:82, 1981.

6. DALBY, J. (Programmer): BMD8V - Analysis of Variance, Statistical Reseatch Laboratory, University of Michigan, Ann Arbor, MI, 1968.

7. GUENTHER, W.C.: Analysis of Variance, Englewood Cliffs, NJ: Prentice-Hall, Inc., 1964.

8. POWERS, J.M.; FARAH, J.W.; and CRAIG, R.G.: Modulus of Elasticity and Strength Properties of Dental Cements, JADA 92:588, 1976

9. POWERS, J.M. and FARAH, J.W.: Apparent Modulus of Elasticity of Dental Amalgams, $J$ Dent Res 54(4):902, 1975.

10. ROBERTS, J.C.; POWERS, J.M.; and CRAIG, R.G.: Fracture Toughness and Critical Strain Energy Release Rate of Dental Amalgam, J Mater Sci 13:965, 1978. 